\title{
MASS DISTRIBUTION AND BULK DENSITY DISTRIBUTION OF INTERPLANETARY DUST
}

\author{
A. HAJDUK \\ Astronomical Institute \\ Slovak. Academy of Sciences \\ 84228 Bratislava \\ Czechoslovakia
}

AESTRACT. Mass distribution of the interplanetary dust is reexamined taking into account bulk density distribution of the dust and larger particles. It can be shown that the mass index of particles depends on the evolutionary stage of the population and changes along the mass scale. The flattening of the mass distribution at the higher mass range may explain the problem of the equilibrium between the source and sink of the interplanetary dust.

\section{MASS DISTRIBUTION OF FAFTICLE FDFULATIONS}

Classical models of meteor stream formation suggested that old showers have lower mass index (s) values because Foynting-Robertson drag and solar radiation pressure gradually eliminate small particles from the stream. It was argued similarly that large particles should dominate in the inner side of the stream. However, these theoretical conclusions have not been confirmed by observations, at least for particles with masses $m \geq 10^{-\delta} \mathrm{kg}$, corresponding to the visual and radar range of detection. As shown recently by Simel (1987), in contrary, showers without an active parent body (e.g. Geminids or Quadrantids) are characterized by higher values of the mass index, implying a lower proportion of larger particles. This is in agreement with the results of spaceprobes, indicating the dominant role of large particies in P/Halley mass production (McDonnell et al., 1987; Hajduk, 1987a) supported also by the improved dust/gas ratios for comet Halley, showing much higher dust contribution (Crifo, 1987; Hajduk, 1987b) than previously reported. Moreover, as shown in the same papers, the mass index is not constant over the mass scale and clearly indicates the superposition of two populations of particles with considerably different mass distribution. This result obtained from spaceborne experiments coincides totaly with results of radar meteor observations showing two separate levels of the mass index for the Halley showers, with values $5=1.8$ and $s=2.2$ (Hajduliova et al., 1987). As it is seen in Figure 1 (A) different particle flux distribution corresponds to these two populations of particles within the shower. Ceplecha (1987) has classified different

A.C. Levasseur-Regourd and H. Hasegawa (eds.), Origin and Evolution of Interplanetary Dust, 331-334.

(C) 1991 Kluwer Academic Publishers, Printed in Japan. 
types of cometary material, having different bulk densities, not necessarily originating in different comets. However the bimodal nature of particle size distributions has been reported also from infrared and optical observations of different comets (Liu and Kimura, 1985).

The question arises, whether the coincidence of the same particle flum and the same abundance of particles with different bulk densities $\gamma_{\rho}=0.75$ and 2.0$)$ at the mass range between $10^{-5} \mathrm{~kg}-10^{-4} \mathrm{~kg}$ is accidental or dependent. (See Figures $1 \mathrm{~A}$ and $1 \mathrm{~B}$. Figure $1 \mathrm{~B}$ is based on Ceplecha"s data quoted above.) The mass distribution of meteor showers, in general, has a maximum mass contribution between the limits of $10^{-6}-10^{-4} \mathrm{~kg}$. Fig. $1 \mathrm{C}$ is constructed for the Halley showers. The caincidence with the flux curves crossing is, of course, not accidental; it shows the meaning of particle populations in the stream: the derived mass contribution of particles of different mass categories is very sensitive to the value of the mass index. We will deal here with the integrated mass index 5 , as defined by Millman (1970) On the balance near the critical value of $S=1$ depends the mass contribution to the stream. In Figure $1 \mathrm{D}$ it is shown that the observed values of 5 for the showers (SH) (Hajdukova et al., 1987) are between the values derived for the cometary halo (H) from space observations (McDonnell et al., 1987; Hajduk and kapisinsky, 1987) and for the large particles from fireball (F) observations (Rendtel and knofel, 1989). The differences in the mass distribution correspond clearly to the age of these populations, as it was shown by Hajduk (1989). As a consequence of a mixture of old and new particle populations, different mass distribution may be observed, depending on the combination of stream filaments met by Earth in a particular return of the shower. Hence we can conclude that showers with an active parent body cannot be characterized by a single mass index value. However, the separation of populations corresponding to the stream structures dyramically bound to possible ejection times, coould be used for the determination of the oldest fraction with the highest value of the mass index.

\section{EFIOSION OF LAFGE PARTICLES}

Classical theories of the stream formation supposed a quick elimination of small particles from the stream by the action Poynting-Robertson effect and solar radiation pressure. The formula of Wyatt and whipple (1950) gives the age of the spiralling particle depending on the particle radius and density and on the orbital parameters. However, the physical erosion processes change drastically along the particle mass scale. As shown by kapisinský (1984), direct light pressure, solar wind corpuscular pressure, Foynting-Robertson effect and other processes have much less effect on the lifetime of particles with $\mathrm{m}>10^{-12} \mathrm{~kg}$ than the destructive processes. The greatest influence on the larger particles is the effect of impact erosion and of corpuscular sputtering. These two effects dominate in the range of meteoroid size particles (Kapišinsky, 1987). (See Figure 1 E). Gron (1987) considers collisional fragmentation to be the dominating process for particles with masses $\mathrm{m} \geqq 10^{-6} \mathrm{~kg}$. The maximum mass contribution in meteor streams comes from $10^{-5+1} \mathrm{~kg}$ and the 

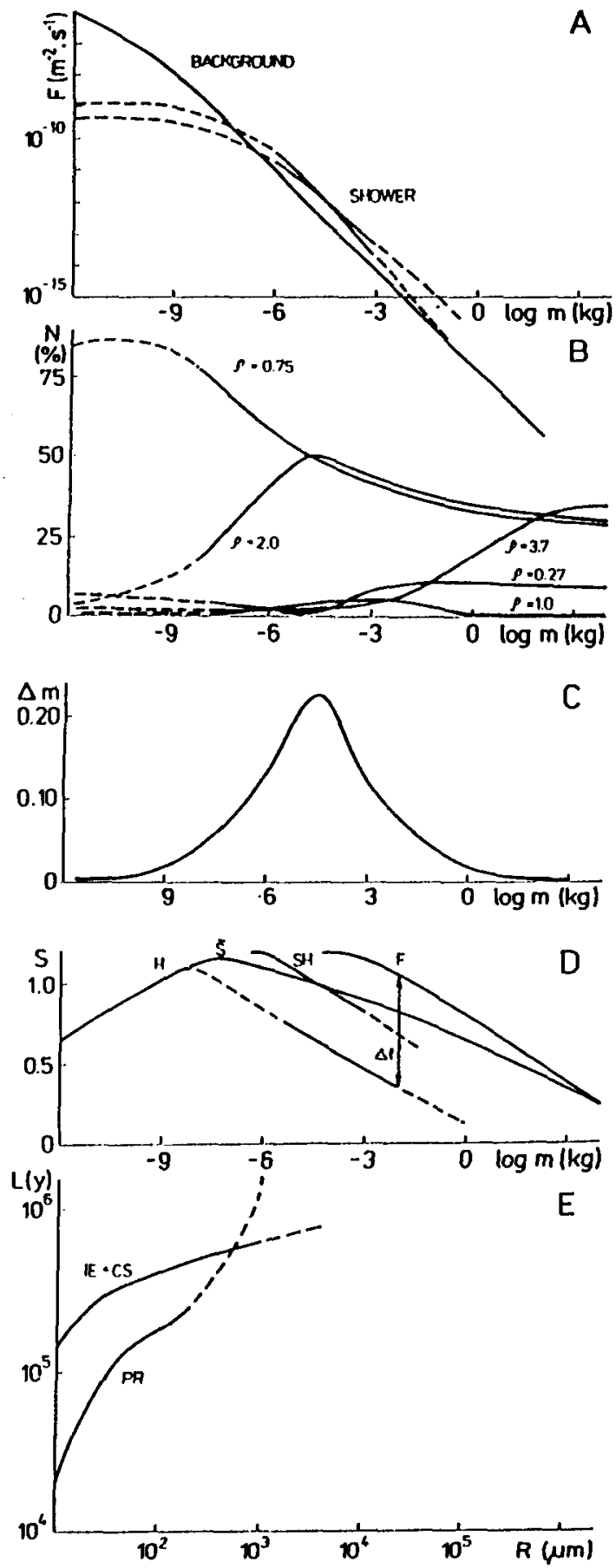

Figure 1

A: Farticle fluk to mass relation for shower and background.

B: Particle bulk density distribution along the mass scale.

C: Mass distribution in meteor shower (Halley showers data).

D: Integrated mass index $\mathbf{S}$ for the halo (H) population (space probes data), Halley showers (SH) (radar observations) and fireballs (F). S denotes the mean values of 5 .

E. Lifetimes of particles due to combined impact erosion (IE) with corpuscular sputtering (CS) compared with PoyntingRobertson (FR) effect. 
maximum mass contribution towards smaller masses. This effect, verifiable from the magnitude distributions of meteors, can then be applied as an independent ageing scale of streams.

References:

Ceplecha, Z. (1987) 'Numbers and masses of different populations of sporadic meteoroids from photographic and television records, in Proc. 10th Europ n Fegional Astronomy Meeting of the IAU Praha 2, 211-215.

Crifo, J. F. (1987) "Are cometary dust mass loss rates deduced from optical emissions reliable?' in Froc. 10th Europ. Regional Astronomy Meeting of the IAU Praha 2, pp. 59-66.

Grun, E. (1987) "Dynamics of interplanetary dust' in Froc. 10th Europ. Regional Astronomy Meeting Fraha 2, pp. 177-178.

Hajduk, A. (1989) "Evolution of cometary debris: physical aspects" "IAU Cal1. 116: Comets in the Post Halley Era, Eamberg, in press.

Hajduk, A. and Kapisinstý, I. (1987) "The evolution of the mass distribution of cometary particles', in Symp. on the Diversity and Similarity of Comets, Arussels, ESA SF-278, pp. 441-444.

Hajdukova, M., Hajduk, A., Cevolani, G., Formiggini, C. (1987) "The P/Halley meteor showers in 1985-1986" Astron. Astrophys. 187, 919-920.

Kapisinstý, I. (1984) 'Nongravitational effects affecting smal 1 meteoroids in interplanetary space", Contrib. Astron. Obs. Skalnat Fleso 12, 99-111.

Kapi sinský, I. (1987) "Double erosion of dust particles", Bull. Astron. Czechosl. 38, 7-12.

Liu, C. F. and Kimura, H. (1985) "On the bimodal nature of the particle-size-distribution function of cometary dust", Marseille Symp. 9-12 July 1984, Froperties and interactionof interplanetary dust, pp. 279-282 (IAU Colloq. No. 85)

MeDonnel1, J. A. M., et al. (1987) "The dust distribution within the inter coma of comet F/Halley 1982i: encounter by Giotto's impact detectors", Astron. Astrophys. 187, 719-741.

Millman F. M. (1970) "Meteor showers and interplanetary dust", Space Fiesearch $X, 260-265$.

Millman, F. M. (1975) 'Dust in the Solar System', The Dusty Universe, MeGraw-Hi11, New York, 185-209

Mukai, T. and Fechtig, H. (198S) "Facking effect of fluffy particles" Planet. Space Sci. 31, 655-658.

Mukai, T., Fechtig, H., Grun, E., and Giese, R. H. (1989) 'Icy particles from comets' Icarus $80,254-266$.

Rendtel, $j$ and knofel, A. (1989) :Analysis of annual and diurnal variation of fireball rates and population index of fireballs from different compilations of visual observations', Bull. Astron. Inst. Caechosl. 40, 53-62.

Simek, M. (1987) "Dynamics and evolution of the structure of five meteor streams", Bul1. Astron. Inst. Caechosl. 38, Bo-91.

Wyatt, S. F. and Whipple, F. L. (1950) "The Poynting-fiobertson effect on meteor orbits", Astrophys. J. 111, 134-141. 